\title{
Quantum Haplodynamics, Dark Matter, and Dark Energy
}

\author{
Harald Fritzsch ${ }^{1,2}$ and Joan Solà ${ }^{2,3}$ \\ ${ }^{1}$ Physik-Department, Universität München, 80333 Munich, Germany \\ ${ }^{2}$ Institute for Advanced Study, Nanyang Technological University, 50 Nanyang Avenue, Singapore 639798 \\ ${ }^{3}$ High Energy Physics Group, Department of ECM and Institut de Ciències del Cosmos, Universitat de Barcelona, \\ Avinguda Diagonal 647, Catalonia, 08028 Barcelona, Spain \\ Correspondence should be addressed to Joan Solà; sola@ecm.ub.edu
}

Received 25 June 2014; Accepted 4 August 2014; Published 31 August 2014

Academic Editor: Frank Filthaut

Copyright (c) 2014 H. Fritzsch and J. Solà. This is an open access article distributed under the Creative Commons Attribution License, which permits unrestricted use, distribution, and reproduction in any medium, provided the original work is properly cited. The publication of this article was funded by SCOAP S $^{3}$

\begin{abstract}
In quantum haplodynamics (QHD) the weak bosons, quarks, and leptons are bound states of fundamental constituents, denoted as haplons. The confinement scale of the associated gauge group $S U(2)_{h}$ is of the order of $\Lambda_{h} \simeq 0.3 \mathrm{TeV}$. One scalar state has zero haplon number and is the resonance observed at the LHC. In addition, there exist new bound states of haplons with no counterpart in the SM, having a mass of the order of $0.5 \mathrm{TeV}$ up to a few $\mathrm{TeV}$. In particular, a neutral scalar state with haplon number 4 is stable and can provide the dark matter in the universe. The QHD, QCD, and QED couplings can unify at the Planck scale. If this scale changes slowly with cosmic time, all of the fundamental couplings, the masses of the nucleons and of the DM particles, including the cosmological term (or vacuum energy density), will evolve with time. This could explain the dark energy of the universe.
\end{abstract}

\section{Introduction}

The standard model (SM) of strong and electroweak interactions may not be the final theory of the universe. The dark matter $(\mathrm{DM})$ and dark energy $(\mathrm{DE})$ are fundamental problems awaiting for an explanation [1-5]. In this paper we consider the possible impact of the chiral gauge theory QHD (quantum haplodynamics [6, 7]) on these problems. QHD is a theory of bound states for all the SM particles and the dark matter particles.

If the QCD and QHD couplings take definite values around the Planck scale and this scale is permitted to slowly change with time, the QCD and QHD couplings should change also with time. Hints that the electromagnetic fine structure constant $\alpha_{\mathrm{em}}$ might change with the cosmic evolution are reported in the literature $[8,9]$. If $\alpha_{\mathrm{em}}$ changes in time, we expect that all fundamental coupling constants change in time, including the gravity constant [10-14]. In particular, let us note that the particle masses could also change in time, such as the proton mass. Different experiments have explored this possibility; see, for example, [15-17] and the reviews $[18,19]$. Since the gravity constant $G_{N}$ determines the Planck mass $M_{P}=G_{N}^{-1 / 2}$, one expects that $M_{P}$ depends also on time and slowly evolves with the cosmic expansion.

The present framework obviously implies a link between gravity and particle physics. We will come back to the cosmological implications after describing the essentials of QHD.

\section{QHD}

In QHD all of the SM particles (except the photon and the gluons) are bound states of the fundamental constituents called haplons, $h$, and their antiparticles. A first model of this type was introduced in 1981 (see [20-25]). Here we extend it and assume that the QHD chiral gauge group is the unitary left-right group $S U(2)_{L} \times S U(2)_{R}$, which we will denote by $S U(2)_{h}$ for short. All species of haplons $h$ are $S U(2)$ doublets; hence each one has two internal states $h_{i}$ represented by the $S U(2)_{h}$ quantum number $i=1,2$. Rotations among these states are performed by the exchange of two sets of massless $S U(2)_{h}$ gauge bosons $\left(X_{L, R}^{r}\right)_{\mu}(r=1,2,3)$ for each chirality.

There are six haplon flavors, two of them are electrically charged chiral spinors $(\chi=\alpha, \beta)$ and four are charged scalars 
TABLE 1: Quantum numbers of the six haplons: spin $(s)$, electric charge $Q$ (in units of $|e|$ ), and corresponding representations of $S U(3)_{c}$ and $S U(2)_{h}$.

\begin{tabular}{ccccc}
\hline & $s$ & $Q$ & $S U(3)_{c}$ & $S U(2)_{h}$ \\
\hline$\alpha$ & $1 / 2$ & $+1 / 2$ & 1 & 2 \\
$\beta$ & $1 / 2$ & $-1 / 2$ & 1 & 2 \\
$\ell$ & 0 & $+1 / 2$ & 1 & 2 \\
$c_{k}$ & 0 & $-1 / 6$ & 3 & 2 \\
\hline
\end{tabular}

$S$. One scalar $(\ell)$ has electric charge $(+1 / 2)$ and carries leptonic flavor. The other three scalars have charge $(-1 / 6)$ and carry color: $c_{k}=R, G, B$ ("red, green, and blue"). In Table 1 we indicate the relevant quantum numbers.

The complete QHD gauge group is $S U(3)_{c} \times S U(2)_{h} \times$ $U(1)_{\mathrm{em}}$, with the coupling constants $\left(g_{s}, g_{h}, e\right)$. There is a gauge coupling $g_{h}=g_{h}^{L}, g_{h}^{R}$ for each chiral factor in $S U(2)_{h}$. The QHD part of the interaction Lagrangian involving a generic chiral haplon $\chi\left(\chi_{L}\right.$ or $\left.\chi_{R}\right)$ and a scalar haplon $S$ reads

$$
\mathscr{L}_{\text {int }}=\bar{\chi}^{i} \gamma_{\mu} i \mathscr{D}_{i j}^{\mu} \chi^{j}+\left(D_{i j}^{\mu} S^{j}\right)^{*}\left(D_{\mu}^{i j} S_{j}\right)+\cdots,
$$

where $\mathscr{D}_{i j}^{\mu}=\delta_{i j} \partial^{\mu}+i g_{h}^{L, R}\left(X_{L, R}^{r}\right)^{\mu}\left(\sigma_{r} / 2\right)_{i j}$ is the $S U(2)_{h}$ covariant derivative and $\sigma_{r}$ are the Pauli matrices. The other covariant derivative is coincident with the previous one if $S$ is any scalar haplon.

For colored scalar haplons $c_{k}$, however, we have additional terms where the covariant derivative is $D_{i j}^{\mu}=\delta_{i j} \partial^{\mu}+i g_{s} A_{a}^{\mu}\left(\lambda^{a} /\right.$ $2)_{i j}$ and involves the gluons $A_{a}^{\mu}$ and Gell-Mann matrices $\lambda^{a}$. Only the scalar haplons $c_{k}$ interact with the gluons, since these are the only colored constituents. Besides the fine structure constant $\alpha_{\mathrm{em}}=e^{2} / 4 \pi$ there is the strong coupling $\alpha_{s}=g_{s}^{2} / 4 \pi$ and its $S U(2)_{h}$ counterpart $\alpha_{h}=g_{h}^{2} / 4 \pi$, which is also strong at energies $\mu \leqslant \Lambda_{h}$ but asymptotically free well above it. Here $\Lambda_{h}$ denotes generically any of the two confining scales $\Lambda_{h}^{L}$ and $\Lambda_{h}^{R}$ associated with the chiral factors of $S U(2)_{h}$.

From the various haplon flavors the bound states of QHD can be constructed. Only for energies $\mu$ well above $\Lambda_{h}$ these states break down into the fundamental haplons. The weak gauge bosons are $s$-wave bound states of left-handed haplons $\alpha$ and $\beta$ and their antiparticles: $W^{+}=\bar{\beta} \alpha, W^{-}=\bar{\alpha} \beta$, and $W^{3}=(\bar{\alpha} \alpha-\bar{\beta} \beta) / \sqrt{2}$.

The neutral weak boson mixes with the photon (similar to the mixing between the photon and the neutral $\rho$-meson). One obtains the physical $Z$-boson with a mass slightly heavier than the $W$-boson:

$$
\frac{M_{Z}^{2}-M_{W}^{2}}{M_{W}^{2}}=\frac{m^{2}}{1-m^{2}},
$$

where the mixing parameter $m=e F_{W} / M_{W}$ is related to the $W$ decay constant $F_{W}[6,7]$. The confinement scale $\Lambda_{h}^{L}$ for $S U(2)_{L}$ defines the Fermi scale $G_{F}^{-1 / 2} \sim 0.3 \mathrm{TeV}$ and the size of the weak gauge bosons of the SM.

Owing to the $S U(2)_{L} \times S U(2)_{R}$ chiral structure of QHD, besides the three observed weak bosons there are also vector bosons that are coupled to the right-handed leptons and quarks. We assume that the right-handed confining scale $\Lambda_{h}^{R}$ is higher so that the masses of the new vector bosons lie well above $1 \mathrm{TeV}$. They might be observed in the new experiments at the LHC. The observed scalar resonance is a $p$-wave excitation of the $Z$. Here we will not discuss these aspects of QHD in detail [6,7].

The leptons and quarks are themselves bound states. They are composed of a chiral haplon $(\alpha$ or $\beta)$ and a scalar haplon: $\ell$ for leptons and $c_{k}$ for quarks. The electron and its neutrino have the structure $v=(\alpha \bar{\ell})$ and $e^{-}=(\beta \bar{\ell})$, which is consistent with the quantum numbers of Table 1. Similarly, the up and down quarks (with $c_{k}$ color) are given by $u=\left(\alpha \bar{c}_{k}\right)$ and $d=$ $\left(\beta \bar{c}_{k}\right)$.

In QHD the first generation of leptons and quarks describes the ground states of the fermion-scalar bound states; the second and third generation must be dynamical excitations. Likewise the $c$-quark and $t$-quark families are excitations of the first quark generation. Compared to the QHD mass scale the masses of the observed leptons and quarks are essentially zero.

The outcome is an effective theory equivalent to the electroweak SM in good approximation. However, new matter content is predicted. In particular, the simplest neutral bound state of the four scalars with haplon number $\mathscr{H}=4$ is a stable color singlet spinless boson: $D=(l R G B)$. It is stable due to haplon number conservation, which is similar to the conservation of baryon number.

\section{A New Dark Matter Candidate}

The mass of the $D$-boson is expected to be in the region of a few $\mathrm{TeV}$. It can be produced together with its antiparticle by the LHC accelerator, and it can be observed by the large missing energy. We interpret it as the particle providing the DM in the universe. The properties of this DM particle are similar to a "weakly interacting massive particle" (WIMP) but it can be much more elusive concerning the interactions with nuclei.

After the Big Bang the universe is filled not only with a gas of quarks and antiquarks but also with a relativistic gas of $D$-bosons and the corresponding antiparticles, which annihilate into other particles. Due to the CP violation there is an asymmetry in the number of $D$-bosons and anti- $D$ bosons. After freeze-out, at temperatures roughly $~ 1 / 20$ of the $D$-boson mass, the $D$-particles abandon the equilibrium. A relic density remains today, a gas of $D$-bosons, forming the dark matter. If we use the average density of matter in our galaxy, we find that there should be $\mathcal{O}(100) D$-particles per cubic meter.

In contrast to more conventional WIMPs, the QHD candidate for DM can escape more easily the recent, highly restrictive bounds obtained from scattering of DM particles off nuclei [26]. It is not difficult to estimate the cross-section for the $D$-boson off a nucleon $\mathcal{N}$ (of mass $m_{\mathcal{N}}$ ). It should be of order

$$
\sigma_{D, \mathcal{N}} \sim f_{D}^{2} \frac{\alpha_{h}^{2}}{\Lambda_{h}^{4}} m_{\mathcal{N}}^{2} \sim f_{D}^{2} \alpha_{h}^{2} G_{F}^{2} m_{\mathcal{N}}^{2}
$$


where $G_{F}^{-1 / 2} \sim \Lambda_{h} \sim 300 \mathrm{GeV}$ according to our definition of Fermi's scale in QHD. Here $f_{D}$ is the dimensionless form factor of the $D$-meson, which describes the confinement of the haplons by the $S U(2)_{h}$ strong gauge force. All QHD bound states have a form factor, which is of order one only for gauge boson mediated interactions, which are described by the exchange of weak bosons $\left(M_{W}^{2} \lesssim \Lambda_{h}^{2}\right)$. For a deeply bound state as $D$, however, we rather expect $f_{D} \sim \Lambda_{h}^{2} / B_{D}^{2} \ll 1$, where $B_{D}$ is the characteristic binding energy scale.

In QCD the proton mass is given by a few times the value of the confining scale $\Lambda_{\mathrm{QCD}}$, with only a tiny contribution from the quark masses. Similarly, in QHD the masses of the bound states are proportional to $\Lambda_{h}$, although here the spectroscopy is richer and the proportionality factor is bigger for the more deeply bound states. Whereas for a weak boson such factor is of order one, in the case of the DM candidate $D=(l R G B)$ it can be much larger. This way we can explain the small cross-section of the DM particles with ordinary matter. For $B_{D}>\mathcal{O}(10) \mathrm{TeV}$ the scattering cross-section of $D$-bosons off nucleons, (3), can be approximately reduced to the level of $\sim 10^{-45} \mathrm{~cm}^{2}$ and become roughly compatible with the current bounds [26]. Let us note that, for energy scales $\mu>10 \mathrm{TeV}$, we have $\alpha_{h}(\mu)=\mathcal{O}(0.1)$ owing to the asymptotic freedom of the haplon interaction constant (cf. next section). One cannot be more precise at this point because at present we cannot work out the details of the form factor $f_{D}$, and therefore (3) is only indicative of the kind of result that could be obtained. The correct order of magnitude could be reached if the bound state associated with DM in this model is very tightly bound and massive. For the exceptional fourhaplon state $D=(l R G B)$ this situation should be regarded, in principle, as possible. We believe that as a possibility it is worthwhile to take into account, still more considering that the present bounds from direct searches put very stringent constraints on virtually every DM candidate, including the more familiar ones from more conventional extensions of the SM.

\section{Unification at the Planck Scale}

The QHD, QCD, and QED couplings might unify at the Planck scale. We can check it at one-loop level, starting from their low-energy values and using the renormalization group equations (RGEs) to compute the running of these parameters. For $S U(N)$ groups $(N>1)$ one has [27]

$$
\frac{d \alpha_{i}}{d \ln \mu}=-\frac{1}{2 \pi}\left(\frac{11}{3} N-\frac{2}{3} n_{f}-\frac{1}{6} n_{s}\right) \alpha_{i}^{2} \equiv-\frac{1}{2 \pi} b_{N} \alpha_{i}^{2} .
$$

Here we have $\alpha_{i}=\alpha_{h}, \alpha_{s}\left(n_{f}\right.$ and $n_{s}$ are the number of fermion flavors and scalars). For the $U(1)$ coupling $\alpha_{\mathrm{em}}$ we have a formula similar to (4), but in this case

$$
b_{1}=-N_{h}\left(\frac{4}{3} \sum Q_{f}^{2}+\frac{1}{3} \sum Q_{s}^{2}\right) \text {. }
$$

Here $N_{h}=2$ for $S U(2)_{h}$. The electric charges $Q$ are defined in Table 1. For energies below $\Lambda_{h}$ we have to replace $N_{h}$ in $b_{1}$ with $N_{c}=3$ (or 1 ) and use the electric charges of the quarks (leptons) rather than those of the haplons.
TABLE 2: The QED, QCD, and QHD fine structure constants $\alpha_{i}=$ $g_{i}^{2} / 4 \pi$ at the $Z$-pole scale $\mu_{0}=M_{Z}$, at an intermediate high energy scale $\mu_{1}=2 \mathrm{TeV}$ (around the haplon continuum threshold), and at the Planck energy $M_{P} \sim 1.2 \times 10^{19} \mathrm{GeV}$, for the $S U(2)_{h}$ chiral gauge group of QHD.

\begin{tabular}{lccc}
\hline & $\mu_{0}$ & $\mu_{1}$ & $M_{P}$ \\
& $M_{Z}$ & $2 \mathrm{TeV}$ & $10^{19} \mathrm{GeV}$ \\
\hline$\alpha_{\mathrm{em}}$ & 0.007816 & 0.008092 & 0.008727 \\
$\alpha_{s}$ & 0.1184 & 0.08187 & 0.01370 \\
$\alpha_{h}$ & - & 0.62 & 0.030 \\
\hline
\end{tabular}

For the fine structure constant $\alpha_{\mathrm{em}}$ we extrapolate its value from low energies to the Planck scale $M_{P} \simeq 1.22 \times 10^{19} \mathrm{GeV}$. At the mass of the $Z$-boson we have $\alpha_{\mathrm{em}}^{-1}\left(M_{Z}\right)=127.94 \pm$ 0.014 . From the mass scale of the $Z$-boson, $\mu=M_{Z}$, until a scale well above $\Lambda_{h}$, say $\mu \sim 2 \mathrm{TeV}$, we use the RGE, taking into account the charges of the three charged leptons and of the five quarks, not including the top quark: $\alpha_{\mathrm{em}}^{-1}(2 \mathrm{TeV})=$ 123.57. From $\sim 2 \mathrm{TeV}$ up to the Planck mass we take into account the electric charges of the two spin- $1 / 2$ haplons and of the four scalar haplons (cf. Table 1). The result is

$$
\alpha_{\mathrm{em}}^{-1}\left(M_{P}\right)=114.58,
$$

equivalently, $\alpha_{\mathrm{em}}\left(M_{P}\right)=0.008727$ (cf. Table 2).

A similar procedure can be followed to compute the QCD coupling constant at various energies. The accurate measurement of this constant at the $Z$-pole yields $\alpha_{s}\left(M_{Z}\right)=$ $0.1184 \pm 0.0007$. At the Fermi scale $\Lambda_{h} \sim 0.3 \mathrm{GeV}$ we find $\alpha_{s}\left(\Lambda_{h}\right)=0.1010$. Well above $1 \mathrm{TeV}$ up to the Planck scale the renormalization proceeds via haplon pairs (as indicated in Table 2).

For the $S U(2)_{h}$ group, we focus here on the left-handed sector and assume $\Lambda_{h} \simeq 0.3 \mathrm{TeV}$. We find, for example, $\alpha_{h}(2 \mathrm{TeV})=0.62$, and eventually at the Planck energy $\alpha_{h}\left(M_{P}\right) \simeq 0.030$. From Table 2 we see that the three couplings approach each other at the Planck scale. The details of the unification will depend on the particular GUT group and can be affected by Clebsch-Gordan coefficients of $\mathcal{O}(1)$.

We note that $S U(3) \times S U(2)_{L} \times S U(2)_{R} \times U(1)$ is a natural breakdown step for GUT groups such as, for example, $S O(10)$. In our case we do not have spontaneous symmetry breaking (SSB); the breaking is always meant to be dynamical. The complete QHD group can thus be naturally linked to the GUT framework without generating unconfined vacuum energy.

If the three couplings come close at the Planck scale, interesting consequences can be derived in connection to the time variation of the fundamental constants, of which hints in the literature appear quite often $[8,9,15-19]$. Exact unification is not essential; we only require that the three couplings take fixed values at or around $M_{P}$.

\section{Time Evolution of Fundamental "Constants"}

A cosmic time change of Newton's constant $G_{N}$ (and hence of $M_{P}$ ) is conceivable in the same way as one admits a 
possible time change of $\alpha_{\mathrm{em}}[8,9,18,19]$. If the QED, QCD, and QHD coupling constants emerge at the Planck epoch, their primeval values should be very close and not be time dependent. Since the Planck energy changes in time, there must be time evolution of the gauge couplings at lower energies, say around the confining scale of the weak bosons, $\Lambda_{h} \sim 300 \mathrm{GeV}$. At the same time the masses of all the particles (including of course the baryons and the $D$-bosons) are forced to slowly evolve with the cosmic expansion since their binding energies are functions of the coupling strengths.

We can estimate the time change of $G_{N}$ in QHD. We use the approximate time variation of $\alpha_{\mathrm{em}}$ suggested in a typical measurement where the current value of the QED coupling is compared with that of a quasar some 12 billion years ago $[8,9]: \Delta \alpha_{\text {em }} / \alpha_{\text {em }}=(-0.54 \pm 0.12) \times 10^{-5}$.

From the RGEs and setting $\mu=M_{P}$ we can obtain the time variation (indicated by a dot) of the Planck scale. Since $b_{1}=-14 / 9$ in this case, we find

$$
\frac{\dot{M}_{P}}{M_{P}}=-\frac{\dot{\alpha}_{\mathrm{em}}\left(M_{Z}\right)}{\alpha_{\mathrm{em}}\left(M_{Z}\right)}\left[\ln \frac{M_{P}}{M_{Z}}+\frac{9 \pi}{7 \alpha_{\mathrm{em}}\left(M_{P}\right)}\right] .
$$

It follows that $\Delta M_{P} / M_{P} \simeq 0.0027$ or $\Delta G / G \simeq-0.0054$.

The time variation of the non-Abelian gauge couplings $\alpha_{i}$ (i.e., $\alpha_{s}$ and $\alpha_{h}$ ) at an arbitrary scale $\mu$ below $M_{P}$ is also determined:

$$
\frac{\dot{\alpha}_{i}(\mu)}{\alpha_{i}(\mu)}=\frac{\dot{M}_{P}}{M_{P}}\left[-\ln \frac{M_{P}}{\mu}+\frac{2 \pi}{b_{N} \alpha_{i}\left(M_{P}\right)}\right]^{-1},
$$

where $b_{N}$ was defined in (4).

Since $\dot{M}_{P} / M_{P}$ is fixed from (7), the above equation enables us to compute the cosmic time variation of the QCD and QHD couplings within the last 12 billion years at any desired energy well above $\Lambda_{h}$, for example, at $\mu_{1}=2 \mathrm{TeV}$ (cf. Table 2):

$$
\frac{\Delta \alpha_{s}}{\alpha_{s}} \simeq 1.1 \times 10^{-4}, \quad \frac{\Delta \alpha_{h}}{\alpha_{h}} \simeq 6.3 \times 10^{-4} .
$$

Using the definition of the corresponding confining scales $\Lambda_{i}$ (namely, $\Lambda_{\mathrm{QCD}}, \Lambda_{h}$ ) we can check from the above formulas that their cosmic time evolution [10] is renormalization group invariant and is directly tied to the cosmic evolution of $M_{P}$ itself:

$$
\frac{\dot{\Lambda}_{i}}{\Lambda_{i}}=\frac{\dot{\alpha}_{i}(\mu)}{\alpha_{i}(\mu)} \frac{2 \pi}{b \alpha_{i}(\mu)}=\frac{\dot{M}_{P}}{M_{P}}
$$

Numerically, $\Delta \Lambda_{i} / \Lambda_{i} \simeq 3 \times 10^{-3}$ for the indicated period.

\section{QHD and Dark Energy}

The unification of the QCD and QHD couplings can have nontrivial significance for the combined framework of particle physics and general relativity (GR). It suggests cosmic evolution of all the masses in the universe, both of the nuclei and of the DM particles. This can be perfectly compatible with GR.
In order to preserve the Bianchi identity that is satisfied by the Einstein tensor of the gravitational field equations $\left(\nabla^{\mu} G_{\mu \nu}=0\right)$, the time evolution of the masses can be compensated for by the time variation of one or more fundamental gravitational parameters, typically the gravitational constant $G_{N}$, or the cosmological constant $\Lambda$, or both [10-14].

With the help of the Friedmann-Lemaitre-RobertsonWalker (FLRW) metric one finds that the most general local conservation law preserving the Bianchi identity, corresponding to an isotropic and homogeneous dust matter fluid with density $\rho_{m}$, reads

$$
\frac{G_{N}^{\prime}}{G_{N}}\left(\rho_{m}+\rho_{\Lambda}\right)+\rho_{m}^{\prime}+\rho_{\Lambda}^{\prime}+\frac{3}{a} \rho_{m}=0,
$$

where $a$ is the scale factor and the primes denote derivatives of the various quantities with respect to it. $\rho_{\Lambda}$ is the vacuum energy density.

From (11) we can understand how a theory of bound states at low energies can lead to general evolution of the vacuum energy density $\rho_{\Lambda}$ and Newton's coupling $G_{N}$ in combination with the particle masses.

Let us note that although many of the studies that motivated the possibility of having variable fundamental constants of Nature were mainly focused on possible time variation of the fine structure constant from QSO absorption spectra $[8,9]$, subsequent investigations admitted the possibility of a time variation of the particle masses, for example, the proton mass [15-17]. The predicted time evolution of the particle masses in QHD can be parameterized as $\rho_{m} \sim a^{-3(1-\nu)}[10]$, where the presence of $|\nu| \ll 1$ denotes a very small departure from the standard conservation law $\sim a^{-3}$. Such a departure is not viewed here as a loss or an excess in the number of particles in a comoving volume (beyond the normal dilution law), but rather as a change in the value of their masses. Models with anomalous matter conservation laws of the above type have been carefully confronted with the precise cosmological data on distant supernovae, baryonic acoustic oscillations, structure formation, and CMB anisotropies, and one finds the upper bound $|\nu| \lesssim \mathcal{O}\left(10^{-3}\right)$ [28-30].

The anomalous matter conservation law $\rho_{m} \sim a^{-3(1-\nu)}$ implies via (11) that a dynamical response will be generated from the parameters of the gravitational sector, $G_{N}$ and $\rho_{\Lambda}$. This is how the dark energy can emerge: it is related to the change of the vacuum energy density triggered by the time evolution of all the masses in the universe. While we cannot predict its value, we suggest that it is time dependent, which should be regarded as natural for the vacuum energy density of an expanding universe.

The time variation of the proton mass (and in general of all masses) within the aforementioned parameterization is approximately given as follows:

$$
\left|\frac{\dot{m}_{p}}{m_{p}}\right| \simeq 3|\nu| H
$$


Notice that the index $v$ need not be universal. Here for simplicity we mention only the case of the proton [10]. The corresponding change of the vacuum energy density reads

$$
\left|\frac{\dot{\rho}_{\Lambda}}{\rho_{\Lambda}}\right| \simeq-3|\nu| \frac{\Omega_{m}^{0}}{\Omega_{\Lambda}^{0}} H
$$

where $\Omega_{m}^{0}, \Omega_{\Lambda}^{0}$ are the current cosmological parameters associated with matter and vacuum energy. Similarly, if the gravitational constant can change with time, we expect [10]

$$
\left|\frac{\dot{G}}{G}\right| \lesssim|\nu| H .
$$

Using the current value of the Hubble parameter as a reference, $H_{0}=1.0227 \mathrm{~h} \times 10^{-10} \mathrm{yr}^{-1}$, where $h \simeq 0.70$, and the mentioned limit $|\nu| \lesssim \mathcal{O}\left(10^{-3}\right)$, we find that the time variations of the above parameters are at most of order $\lesssim 10^{-13} \mathrm{yr}^{-1}$. These changes are of course only indicative since $v$ could be smaller, but at that level they are approximately within the present bounds. Such bounds are obtained from many sources and usually with large errors [18, 19]. Let us mention that a new generation of high precision lab experiments could reach the level $\lesssim 10^{-14} \mathrm{yr}^{-1}$ [31]. In that case they might be sensitive to the possible time variations, if they are there.

One cannot exclude a priori that various sorts of effects are involved at the same time. In such case the results can be more difficult to interpret and may require the use of more than one observable. In general this situation might enforce some reinterpretation of the previous observations, in which more emphasis is given to the variation of one constant with respect to another. For example, it is interesting to note that in the context of conventional GUTs one finds that if the $\Lambda_{\mathrm{QCD}}$ scale (and hence the proton mass) changes with time, such change would be significantly larger (by more than one order of magnitude) than the possible time variation of the fine structure constant-see $[10,32,33]$. At the end of the day the most important feature is that a positive observational effect (irrespective of the various sources of time variation concurring in it) would signal the new qualitative fact that the constants of Nature may be varying.

Finally, we should also mention that the models (11) where the variable vacuum energy and gravitational constant can be linked to the variation of the particle masses are compatible with the primordial nucleosynthesis bounds on the chemical species. The important constraint to be preserved here is that the vacuum energy density remains sufficiently small as compared to the radiation density at the time of nucleosynthesis. At the same time, potential variations of the gravitational constant should also be moderate enough to avoid a significant change in the expansion rate at that time. Once more one can show that these situations are under control provided the limit $|\nu| \lesssim \mathcal{O}\left(10^{-3}\right)$ is fulfilled $[30,34,35]$. Under these circumstances the abundances of primordial chemical elements remain essentially the same.

\section{Conclusions}

The framework we have outlined here proposes a new formulation of GUT's involving gravity ab initio (in contrast to more conventional formulations). It also proposes a new candidate for the dark matter that is not in conflict with the recent, highly restrictive bounds for the scattering of DM particles off nuclei. The neutral and stable $D$-boson is the particle of the dark matter.

QHD is not based on the conventional SSB mechanism and it does not lead to a large contribution to the cosmological term. The DE appears here as the tiny (but observable) dynamical change of the vacuum energy density of the expanding background and hence is a part of the generic response of GR to the cosmic time variation of the masses of all the stable baryons and dark matter particles in the universe.

These ideas can be tested by future astrophysical and laboratory tests in quantum optics, which are expected to detect potential proton mass variations of order $\leqslant 10^{-14} \mathrm{yr}^{-1}$ [31], hence at the level of the expected running of the cosmological parameters.

\section{Conflict of Interests}

The authors declare that there is no conflict of interests regarding the publication of this paper.

\section{Acknowledgments}

Harald Fritzsch thanks the BKC excellence programme and the Department of. ECM, University of Barcelona, for hospitality and support. Joan Solà is supported in part by MICINN, DEC, and CPAN. The authors are both grateful to the Institute for Advanced Study at the Nanyang Technological University in Singapore for hospitality and support.

\section{References}

[1] S. Weinberg, "The cosmological constant problem," Reviews of Modern Physics, vol. 61, no. 1, pp. 1-23, 1989.

[2] V. Sahni and A. Starobinsky, "The case for a positive cosmological Lambda-term," International Journal of Modern Physics A, vol. 9, pp. 373-444, 2000.

[3] T. Padmanabhan, "Cosmological constant-the weight of the vacuum," Physics Reports, vol. 380, no. 5-6, pp. 235-320, 2003.

[4] P. J. E. Peebles and B. Ratra, "The cosmological constant and dark energy," Reviews of Modern Physics, vol. 75, no. 2, pp. 559606, 2003.

[5] E. J. Copeland, M. Sami, and S. Tsujikawa, "Dynamics of dark energy," International Journal of Modern Physics D, vol. 15, article 1753, no. 11, 2006.

[6] H. Fritzsch, "The size of the weak bosons," Physics Letters B, vol. 712, pp. 231-232, 2012.

[7] H. Fritzsch, "Composite weak bosons, leptons and quarks," Modern Physics Letters A, vol. 26, no. 31, pp. 2305-2312, 2011.

[8] M. T. Murphy, J. K. Webb, and V. V. Flambaum, "Further evidence for a variable fine-structure constant from Keck/HIRES 
QSO absorption spectra," Monthly Notices of the Royal Astronomical Society, vol. 345, no. 2, pp. 609-638, 2003.

[9] H. Rahmani, N. Maheshwari, and R. Srianand, "Constraining the variation in fine-structure constant using SDSS DR8 QSO spectra," http://xxx.tau.ac.il/abs/1312.5324.

[10] H. Fritzsch and J. Solà, "Matter non-conservation in the universe and dynamical dark energy," Classical and Quantum Gravity, vol. 29, no. 21, Article ID 215002, 25 pages, 2012.

[11] J. Solà, "Cosmological constant and vacuum energy: old and new ideas," Journal of Physics: Conference Series, vol. 453, Article ID 012015, 2013.

[12] J. Solà, "Cosmologies with a time dependent vacuum," Journal of Physics: Conference Series, vol. 283, no. 1, Article ID 012033, 2011.

[13] J. Solà, "Dark energy: a quantum fossil from the inflationary Universe?" Journal of Physics A: Mathematical and Theoretical, vol. 41, no. 16, Article ID 164066, 2008.

[14] J. Solà, "Vacuum energy and cosmological evolution," AIP Conference Proceedings, vol. 1606, pp. 19-37, 2014.

[15] E. Reinhold, R. Buning, U. Hollenstein, A. Ivanchik, P. Petitjean, and W. Ubachs, "Indication of a cosmological variation of the proton-electron mass ratio based on laboratory measurement and reanalysis of H2 spectra," Physical Review Letters, vol. 96, no. 15, Article ID 151101, 2006.

[16] W. Ubachs and E. Reinhold, "Highly accurate $H_{2}$ lyman and werner band laboratory measurements and an improved constraint on a cosmological variation of the proton-to-eiectron mass ratio," Physical Review Letters, vol. 92, no. 10, 2004.

[17] A. Ivanchik, P. Petitjean, D. Varshalovich et al., "A new constraint on the time dependence of the proton-to-electron mass ratio: analysis of the Q 0347-383 and Q 0405-443 spectra," Astronomy and Astrophysics, vol. 440, no. 1, pp. 45-52, 2005.

[18] J.-P. Uzan, "Varying constants, gravitation and cosmology," Living Reviews in Relativity, vol. 14, p. 2, 2011.

[19] T. Chiba, "The constancy of the constants of nature: updates," Progress of Theoretical Physics, vol. 126, p. 993, 2011.

[20] H. Fritzsch and G. Mandelbaum, "Weak interactions as manifestations of the substructure of leptons and quarks," Physics Letters B, vol. 102, no. 5, pp. 319-322, 1981.

[21] H. Fritzsch and G. Mandelbaum, "The substructure of the weak bosons and the weak mixing angle," Physics Letters B, vol. 109, no. 3, pp. 224-226, 1982.

[22] R. Barbieri, R. N. Mohapatra, and A. Masiero, "Compositeness and a left-right symmetric electroweak model without broken gauge interactions," Physics Letters B, vol. 105, no. 5, pp. 369374, 1981.

[23] H. Fritzsch, D. Schildknecht, and R. Kogerler, "Weak current algebra, composite $\mathrm{W}$ bosons and universality of the weak interactions," Physics Letters B, vol. 114, pp. 157-161, 1982.

[24] L. F. Abbott and E. Farhi, "Are the weak interactions strong?" Physics Letters B, vol. 101, no. 1-2, pp. 69-72, 1981.

[25] T. Kugo, S. Uehara, and T. Yanagida, "Weak bosons as composite gauge fields of hidden symmetries," Physics Letters B, vol. 147, no. 4-5, pp. 321-324, 1984.

[26] LUX Collaboration, D. S. Akerib, H. M. Araujo et al., "First results from the LUX dark matter experiment at the Sanford underground research facility," Physical Review Letters, vol. 112, Article ID 091303, 2014.

[27] T. P. Cheng, E. Eichten, and L. Li, "Higgs phenomena in asymptotically free gauge theories," Physical Review D, vol. 9, no. 8, pp. 2259-2273, 1974.
[28] S. Basilakos, M. Plionis, and J. Solà, "Hubble expansion and structure formation in time varying vacuum models," Physical Review D, vol. 80, no. 8, Article ID 083511, 2009.

[29] J. Grande, J. Solà, S. Basilakos, and M. Plionis, "Hubble expansion and structure formation in the "running FLRW model" of the cosmic evolution," Journal of Cosmology and Astroparticle Physics, vol. 1108, 2011.

[30] S. Basilakos, D. Polarski, and J. Solà, "Generalizing the running vacuum energy model and comparing with the entropic-force models," Physical Review D, vol. 86, Article ID 043010, 2012.

[31] T. W. Hänsch, Privatecommunication.

[32] X. Calmet and H. Fritzsch, "Symmetry breaking and time variation of gauge couplings," Physics Letters B, vol. 540, no. 3-4, pp. 173-178, 2002.

[33] X. Calmet and H. Fritzsch, "A time variation of proton-electron mass ratio and grand unification," Europhysics Letters, vol. 76, no. 6, pp. 1064-1068, 2006.

[34] J. Grande, J. Solà, J. C. Fabris, and I. L. Shapiro, "Cosmic perturbations with running G and Lambda," Classical and Quantum Gravity, vol. 27, Article ID 105004, 2010.

[35] I. L. Shapiro, J. Solà, and H. Štefančić, "Running $G$ and $\Lambda$ at low energies from physics at $M_{X}$ : possible cosmological and astrophysical implications," Journal of Cosmology and Astroparticle Physics, vol. 2005, article 012, 2005. 

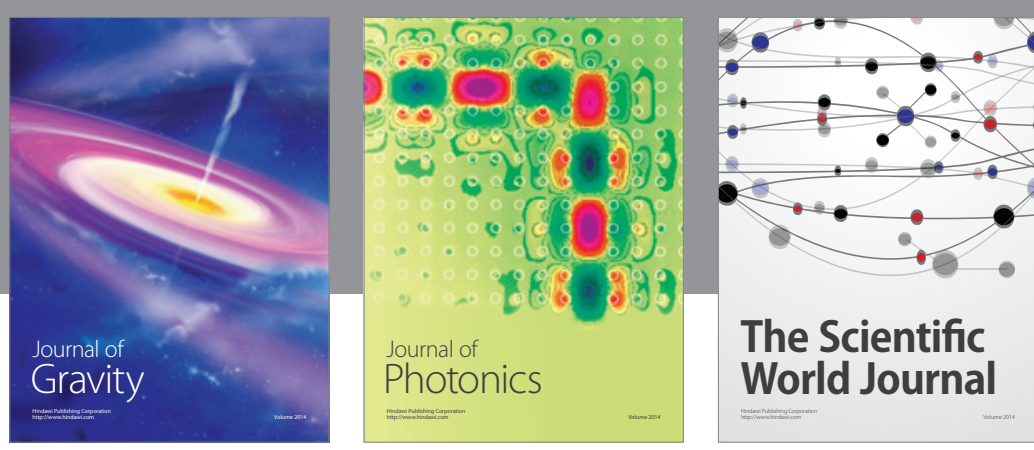

The Scientific World Journal
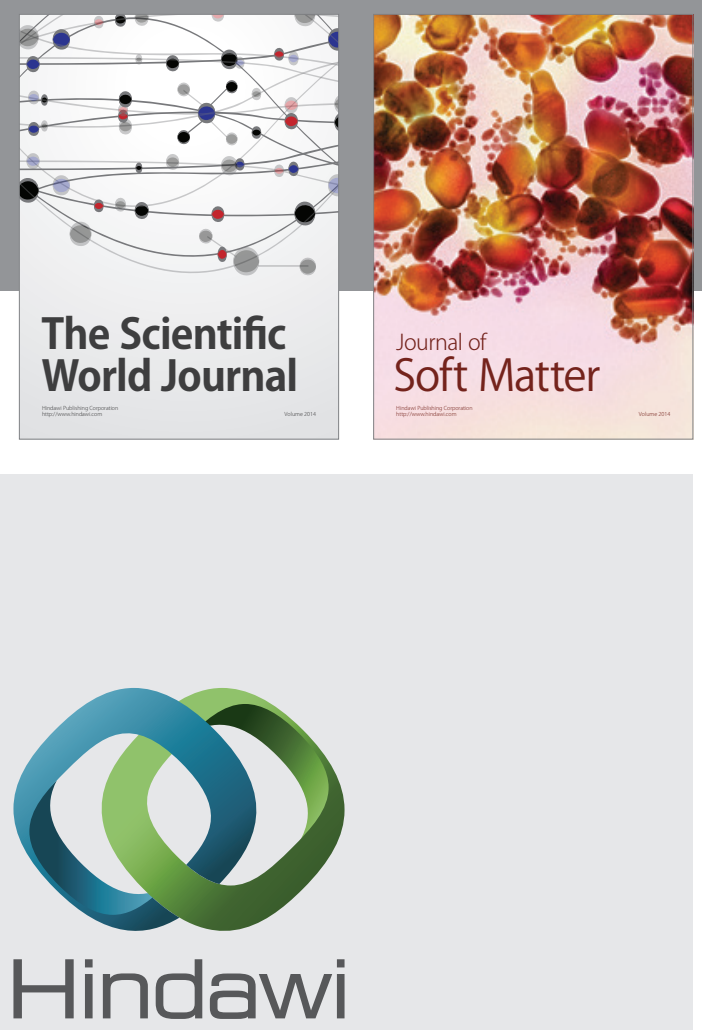

Submit your manuscripts at

http://www.hindawi.com

nternational Journal of

Statistical Mechanics
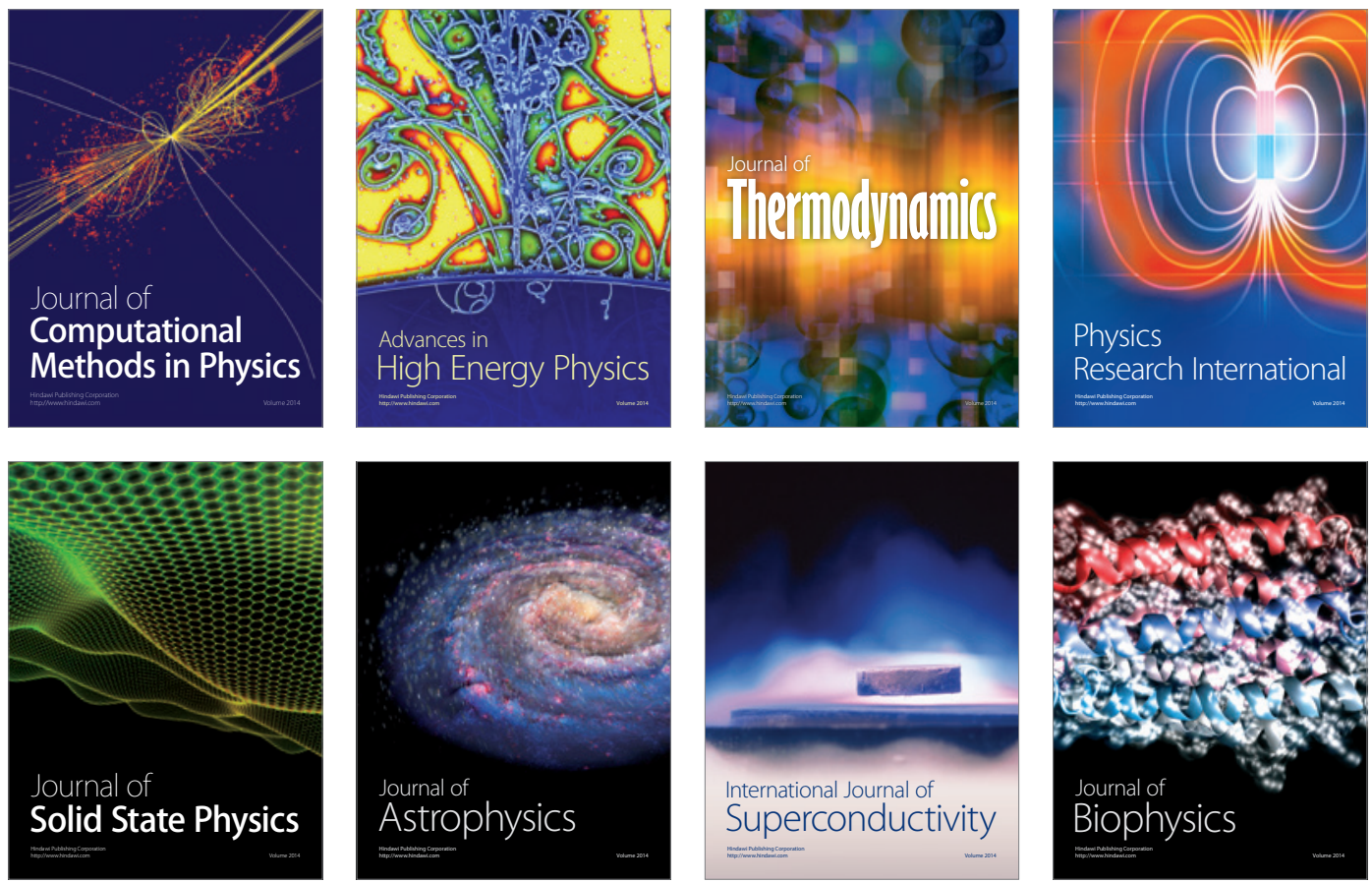
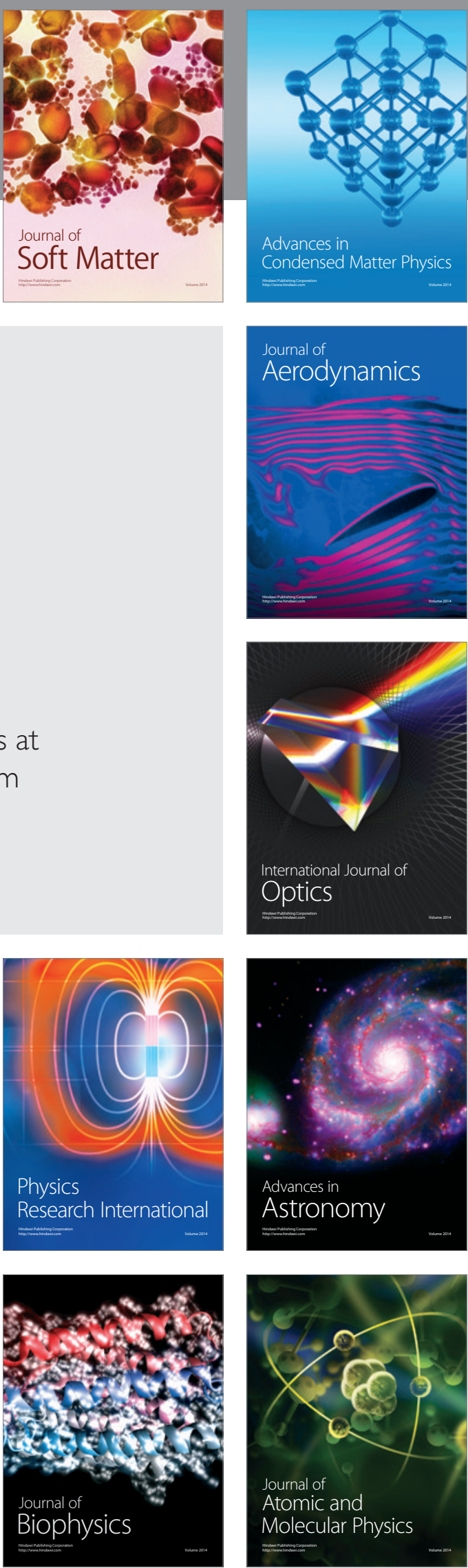